\title{
Model Based System Identification for Hydraulic Deep Drawing Presses
}

\author{
Dipl.-Ing. Tobias Schulze, Prof. Dr.-Ing. Jürgen Weber \\ Chair of Fluid Mechatronic Systems (Fluidtronic) \\ Institute of Fluid Power, TU Dresden, Germany \\ E-mail: tobias.schulze2@tu-dresden.de
}

\begin{abstract}
The paper describes the development of an automated system identification algorithm for the die cushion drive in hydraulic deep drawing presses. The algorithm could successfully be implemented on the drive controller to automatically identify the system parameters of the drive. Main aspect of the paper is the application driven development of an appropriate system model and identification algorithm with its implementation on the drive controller. It could be verified with experiments on a $2500 \mathrm{kN}$ hydraulic deep drawing press. This thorough knowledge of the system model with its parameters shows a high potential to be further evaluated for system diagnosis and could also be used for system simulation and controller design.
\end{abstract}

Key points are the limited processing power of the drive controller and the occurring signal noise. A grey-box system model was chosen and its parameters were identified by means of a recursive least square algorithm. The implementation on the drive controller required adaptions due to restricted cycle time and additional signal processing to reduce noise that will also be discussed in the paper.

Keywords: industrial hydraulics, hydraulic deep drawing press, system identification, system simulation, grey box model, valve control, parameter estimation, orthogonal correlation, moving average filter, symmetrical derivative

\section{Introduction}

The requirements on modern industrial hydraulics are rising. The operator sets highest demands on energy efficiency, best dynamic and static behavior, highest reliability and even requests new functionality. The die cushion in deep drawing presses is a typical application for modern servo hydraulics as it offers a precise adjustability of the counter-holding force on the blank. With velocities of up to $300 \mathrm{~mm} / \mathrm{s}$ and cylinder forces of more than $1000 \mathrm{kN}$ the dynamic behavior needs to be most accurate. Modern blank materials, the raising cushion speeds and more complex tool geometries set an even higher demand on the die cushion. In addition the operator requests low set-up time and minimal service requirements.

An extensive and thorough set-up process and a time consuming maintenance scheme with qualified and experienced technical staff is currently necessary to fulfil these demands $(/ 1 /, / 2 /, / 3 /)$. The intensive set-up process is required for every newly installed deep drawing press and also after every modernization of the hydraulic system.

Model based system optimization methods offer the possibility to support with the set up process and even to increase the quality of the hydraulic drive. But on the other hand those simulation models and optimization methods require a thorough knowledge of the system and its parameters. This is where the method of automated parameter estimation steps in providing a possible solution to identify the system parameters within a few seconds.

The literature offers a high variety of system identification methods $(/ 4 /, / 5 /, / 6 /)$ out of which the most suitable for the hydraulic die cushion drive is to be chosen. Jelali and Kroll /7/ proved the feasibility of system identification for hydraulic drives in an experimental environment. However, the implementation within the drive controller for an industrial machine has not been realized yet.

The use of the current parameters and system model from the identification algorithm are versatile. They can be evaluated for condition monitoring, for fault diagnosis, to increase reliability and to reduce the required time for manual servicing. They can also be used for system simulation and controller design to reduce the set-up time and ensure a high control quality of the hydraulic drive. Several published works show that a model based controller design can be based on an identified system model and deliver an improved control accuracy. The identification algorithm is therefore an important step towards an improved system performance.

The aim of the presented paper is to develop and implement a parameter estimation algorithm within the machine controller of the hydraulic die cushion system. This allows to easily access the current set of parameters by an identification process or even during regular application of the machine. 


\section{Test setup}

The research was conducted on an hydraulic deep drawing press that is available at the Institute of Fluid Power at the TU Dresden (see fig. 1). It features a hydraulic slide with a maximum force of $2500 \mathrm{kN}$ and a modern hydraulic four-point die cushion with a total force of $1000 \mathrm{kN}$. The press can be equipped with different tools, such as a rectangular pan tool and a round cup tool for deep drawing, a cutting tool and a test block that allows the press to work at any possible operating point up to maximum force and speed. All experiments presented in this paper were conducted with this test block representing a deep drawing tool. The deep drawing press is equipped with several additional sensors for hydraulic, mechanic, and electric signals as well as a tailor made measuring system.

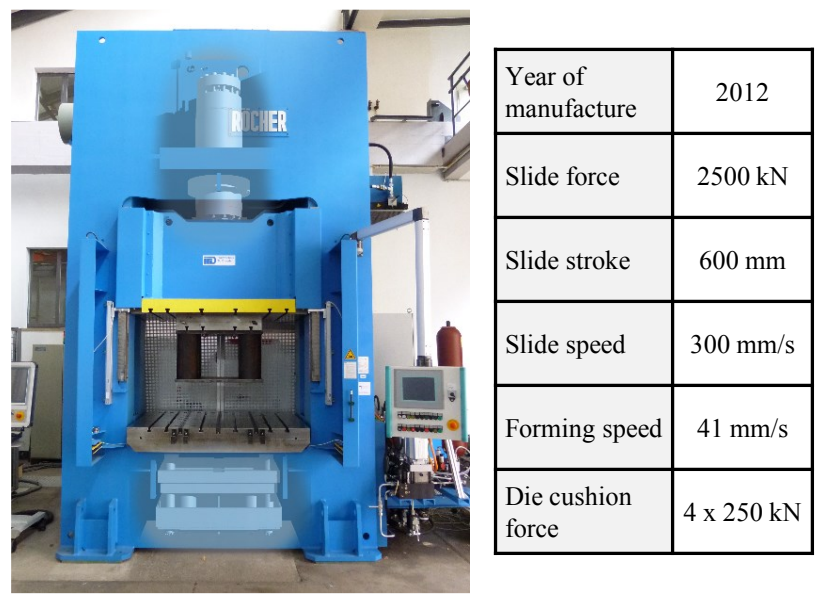

Figure 1: Hydraulic deep drawing press at the IFD

For the hydraulic die cushion, a model based system identification algorithm will be implemented on its drive controller. The controller, a MOOG Servo Drive MSD, features a 32-bit processor with $400 \mathrm{MHz}$ and $256 \mathrm{MB}$ of RAM. Available machine signals from the die cushion drive are the cylinder position $z_{d c}$, the cylinder pressure in both cylinder chambers $p_{A}$ and $p_{B}$ and the applied valve voltage $U_{v}$ at the servo valve as well as the return signal for the actual valve spool position $y_{v}$ for each of the four cylinders. All machine signals are read with Ether CAT connected IO-modules.

The behavior of the hydraulic die cushion will be further investigated within this paper and its model parameters will be identified. The hydraulic structure of the die cushion is shown in fig. 2 for one of the four die cushion cylinders. They are used for position- as well as force-control of the die cushion as there is no dedicated lifting cylinder. An axialpiston pump with internal and external pressure control provides the necessary volume flow for all four cylinders. The hydraulic layout was adapted to allow for a permanent rapid traverse motion on the cylinder-out movement. The cylinder-in movement is simplified in the way that only the pressure chamber on the piston side is controlled by the servovalve and the pressure chamber on the rod side is directly connected to the axial piston pump. A changing valve position of the servo valve does therefore only affect the pressure in the piston side.

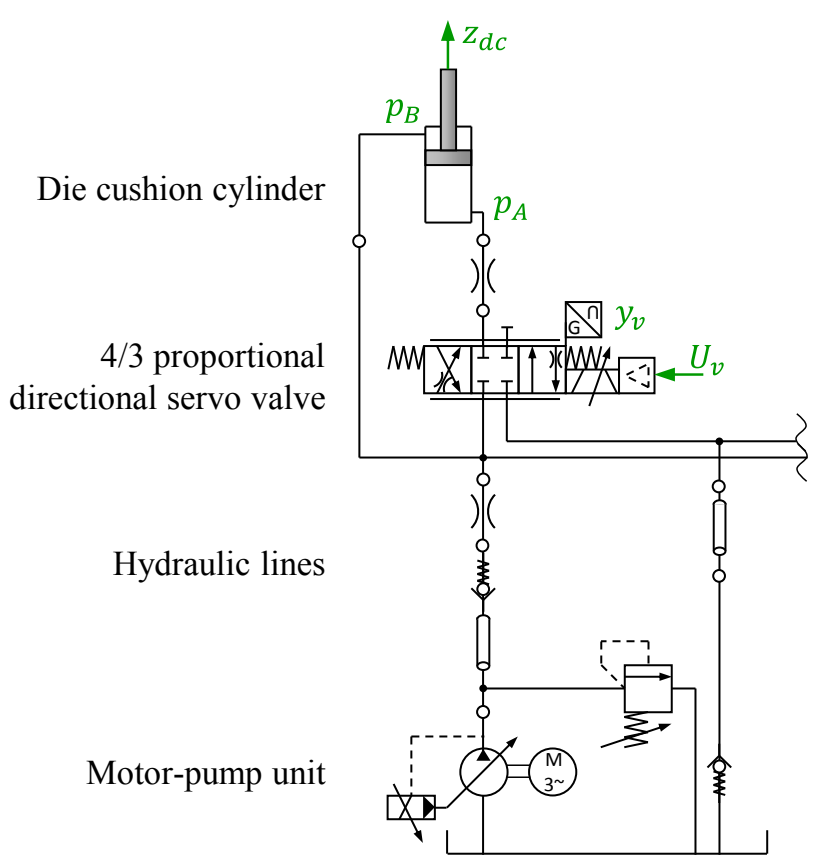

Figure 2: Hydraulic structure of the die cushion and measured system variables

\section{Development of the system model}

The system model forms the basis for the identification algorithm and defines the possibilities for evaluating the model parameters and system characteristics. The system model should behave as close to the real system as possible and allow to describe the real system as accurate as possible. At the same time, the structure and complexity of the system model must meet all requirements for an automated system identification, so the model complexity is limited.

The model derivation was based on the physical principles in order to retain the model parameters with a physical value and equivalent. A so-called white box model with its physically based mathematical equations is a common and widely verified tool to describe the behavior of hydraulic servo systems to a high level of detail. There parameters have a physical meaning and can therefore be checked on fundamental correctness as well as compared with other systems. It also offers a high variety of possibilities for future applications, such as controller design, condition monitoring or fault diagnosis based on the physical system model. However, a pure white-box model with a sufficient accuracy would require a high level of complexity with numerous parameters. Therefore, the physical behavior is described with basic mathematical equations for the fluidic system and is extended with black-box model assumptions in order to achieve a sufficient accuracy with reduced model complexity and fewer model parameters. The system was divided in a model for the fluidic system that is described as a white-box model and the valve dynamic as a black-box model. Within this paper, only the state of force control for the die cushion during the process of deep drawing is considered. This is the most relevant state for the die cushion as it controls the drawing process with the applied force and therefore directly influences the quality of the produced part. 
Figure 3 shows the chosen model structure with its (measureable) system variables for hydraulic pressure $\left(p_{t}, p_{A}\right.$, $p_{B}$ and $p_{0}$ ), servovalve voltage $U_{v}$ and -position $y_{V}$ and the cylinder position $z_{d c}$. The system boundaries are the supply pressure $p_{0}$, the oil tank pressure $p_{t}$, the cylinder position $z_{d c}$ and servovalve voltage $U_{v}$. The system and oil tank pressure were experimentally verified to be constant at approximately $p_{t / 0} \approx 0$ bar. The cylinder position is determined by the ram of the deep drawing press, which is why the cylinder speed $\dot{z}_{d c}$ is assumed as a constant value.

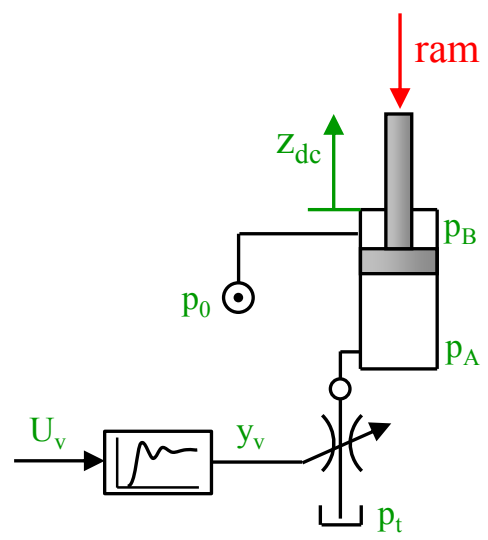

Figure 3: Reduced system model for one die cushion drive cylinder

The differential equations of the hydraulic model are:

$$
\begin{gathered}
\dot{p}_{A}=\frac{K^{\prime}}{V_{0}+A_{A} \cdot z_{d c}}\left(-A_{A} \cdot \dot{z}_{d c}-q_{A}\right) \\
Q_{A}=\operatorname{sign}\left(p_{A}-p_{t}\right) \cdot \frac{Q_{v, n o m}}{\sqrt{\Delta p_{\text {nom }}}} \cdot \sqrt{\left|p_{A}-p_{t}\right|} \cdot\left(y_{v}-y_{0 v}\right)
\end{gathered}
$$

And respectively for the valve dynamics that is modelled as a 2nd-order PT2-element with an additional dead-time:

$$
\begin{gathered}
U_{v}^{*}=U_{v}\left(t-T_{\text {dead }}\right) \\
\frac{1}{\omega_{0 v}^{2}} \ddot{y}_{v}+\frac{2 D_{v}}{\omega_{0 v}} \dot{y}_{v}+y_{v}=K_{v} \cdot U_{v}^{*}
\end{gathered}
$$

This leads to a model with eight system variables $\left(p_{0}, p_{A}, \dot{p}_{A}, p_{B}, Q_{A}, y_{v}, z_{d c}, \dot{z}_{d c}\right)$ and ten parameters $\left(A_{A}, K^{\prime}, V_{0}, Q_{v, \text { nom }}, \Delta p_{\text {nom }}, y_{0 v}, T_{\text {dead }}, \omega_{0 v}, D_{V}, K_{v}\right)$.

Figure 4 shows the system model as a block diagram:

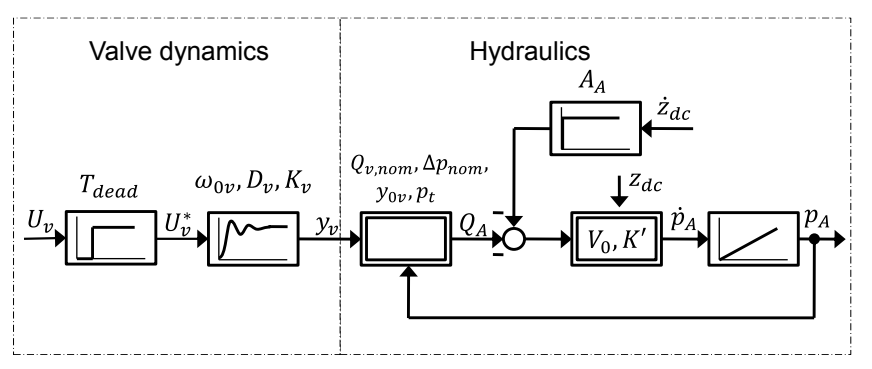

Figure 4: Reduced system model as block diagram

\section{Identification algorithm}

The reduced system model contains 10 parameters that need to be identified. The parameter for the cylinder-area $A_{A}$ can be taken from the data sheet of the hydraulic cylinder easily and with a high accuracy. The nominal pressure drop $\Delta p_{\text {nom }}$ is directly connected to the nominal volume flow $Q_{v, n o m}$ on the servo valve and can therefore be set to a chosen value. The remaining eight parameters need to be identified from the system behavior. An automated identification algorithm should be developed, as a manual process would be highly time consuming.

Isermann describes different methods for system identification with its pros and cons (see /4/, pages $16 \mathrm{f}$ ). The identification highly depends on the chosen model structure and in this particular case must meet all requirements for an online-computation on an industrial PC, respectively machine controller. Therefore a parameter estimation with recursive least square will be implemented for both subsystems, the hydraulics as well as the valve dynamics. The model estimation is capable to identify the parameters of a parametric model, as it was developed in the previous chapter. Furthermore it can be implemented on the machine controller as a recursive algorithm. The requirement on the model structure is to be linear within the parameters with the $(m \times 1)$-sized parameter-vector $\boldsymbol{\theta}$, the $(1 \times n)$-sized systeminput-vector $\boldsymbol{u}$ and the system output $\gamma$ according to equation (5). The number of parameters is represented by $m$ and the number of input values represented by $n$.

$$
\gamma=\boldsymbol{\theta} \cdot \boldsymbol{u}
$$

The task of the parameter estimation algorithm is to determine the model parameters $\theta_{i}$ such that the output error $\varepsilon$ is minimized. The system model is impinged with the same (measured) input $\boldsymbol{u}$ as the real system. The model output can then be calculated with the identified system parameters for each timestep from eq. (5). The output error $\varepsilon$, calculated from the measured output of the real system minus the output of the system model delivers a value for the accuracy of the system model and its parameters. A low value $\varepsilon$ means that the system model and its parameters resemble the behavior of the real system very good. Figure 5 shows the identification process as a schematic figure.

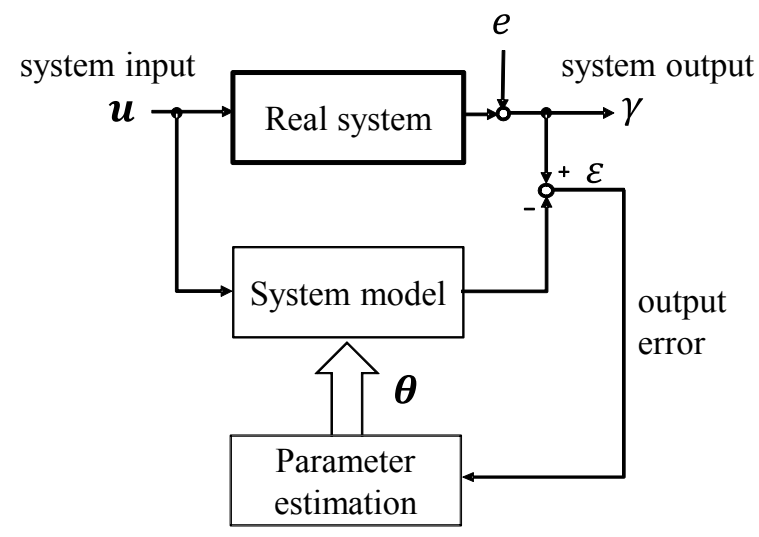

Figure 5: Identification process, schematic 


\subsection{The hydraulic subsystem}

The subsystem for the hydraulic model can be transformed to fit equation (5). Eliminating the volume flow $q_{A}$ in equation (1) with equation (2) leads to the complete system equation that has to be transformed to fit the format in eq.(5). It leads to the following equations:

$$
\begin{aligned}
& \gamma=A_{A} \cdot \dot{z}_{d c} \\
& \boldsymbol{\theta}=\left(\begin{array}{c}
\theta_{1} \\
\theta_{2} \\
\theta_{3} \\
\theta_{4}
\end{array}\right) \\
& \theta_{1}=-\frac{Q_{v, n o m}}{\sqrt{\Delta p_{n o m}}} \\
& \theta_{2}=-\frac{V_{0}}{K^{\prime}} \\
& \theta_{3}=-\frac{A_{A}}{K^{\prime}} \\
& \theta_{4}=\frac{Q_{v, n o m}}{\sqrt{\Delta p_{n o m}}} \cdot y_{0 v} \\
& \boldsymbol{u}=\left(u_{1} u_{2} u_{3} u_{4}\right) \\
& u_{1}=\operatorname{sign}\left(p_{A}-p_{t}\right) \cdot \sqrt{\left|p_{A}-p_{t}\right|} \cdot y \\
& u_{2}=\dot{p}_{A} \\
& u_{3}=z_{d c} \cdot \dot{p}_{A} \\
& u_{4}=\operatorname{sign}\left(p_{A}-p_{t}\right) \cdot \sqrt{\left|p_{A}-p_{t}\right|}
\end{aligned}
$$

The systeminput-vector $\boldsymbol{u}$ is calculated from four measured values: $p_{A}, \dot{p}_{A}, z_{d c}$ and $y$ (with the tank pressure being set to $\left.p_{t} \approx 0\right)$. Furthermore, the system output needs to be calculated from the derivative of the measured cylinder position, respectively the cylinder speed $\dot{z}_{d c}$. All these values are available at the die cushion drive of an hydraulic press and can be evaluated within the machine controller. An additional requirement for the applicability of the chosen algorithm is for the elements of the input vector $\boldsymbol{u}$ to be independent. This can be proven with a singular value decomposition $/ 5 /$. Despite the elements 2 and 3 correlating by the factor $z_{d c}$ and elements 1 and 4 correlating by the factor $y$, the singular value decomposition shows sufficient independency.

The parameter estimation is calculated as a recursive algorithm for each time step $k$. This means that with each time step of the machine controller, the system values (input $\boldsymbol{u}$ and output $\gamma)$ are measured, the model output $(\boldsymbol{\theta} \cdot \boldsymbol{u})$ is calculated and a new set of parameters $\boldsymbol{\theta}$ is determined from the error $\varepsilon$. The parameter estimation algorithm calculates a correcting vector $\boldsymbol{L}$ with each time step to determine the influence of the output error on the new set of parameters:

$$
\begin{gathered}
\boldsymbol{\theta}(k)=\boldsymbol{\theta}(k-1)+\boldsymbol{L}(k) \cdot[\gamma(k)-\boldsymbol{\theta}(k-1) \cdot \boldsymbol{u}(k-1)] \\
\begin{array}{c}
\text { New } \\
\text { Parameter }
\end{array}=\begin{array}{c}
\text { Old } \\
\text { Parameter }
\end{array}+\begin{array}{c}
\text { Correcting } \\
\text { Vector }
\end{array} \cdot\left(\begin{array}{c}
\text { Output } \\
\text { Error }
\end{array}\right)
\end{gathered}
$$

The correcting vector $\boldsymbol{L}$ itself is calculated from the covariance matrix $\boldsymbol{P}$, the system input $\boldsymbol{u}$ and a forgetting factor $\lambda$, see equation (10).

$$
\boldsymbol{L}(k)=\frac{\boldsymbol{P}(k-1) \cdot \boldsymbol{u}(k)}{\lambda+\boldsymbol{u}^{T}(k) \cdot \boldsymbol{P}(k-1) \cdot \boldsymbol{u}(k)}
$$

The covariance matrix is also recalculated for each time step according to equation (11):

$$
\boldsymbol{P}(k)=\frac{1}{\lambda}\left[\boldsymbol{P}(k-1)-\boldsymbol{L}(k-1) \cdot \boldsymbol{u}^{T}(k) \cdot \boldsymbol{P}(k-1)\right]
$$

In order to start the algorithm, initial values for $\boldsymbol{P}(0)$ and $\boldsymbol{\theta}(0)$ need to be predefined as well as an appropriate value for the forgetting factor. The covariance matrix $\boldsymbol{P}$ is of the size $(n \times m)$. Its initial value should be calculated from the identity matrix multiplied with a constant factor $\alpha$ :

$$
\boldsymbol{P}(0)=\alpha \cdot \boldsymbol{I}
$$

The factor $\alpha$ can be chosen from a value $\alpha=100 \ldots 10000$ /4/. For the investigated application of the die cushion drive, a value $\alpha=500$ was chosen. It can be interpreted that the choice of a large value $\alpha$ allows the algorithm to make large changes to the next set of parameters within one single time step at the beginning of the identification $/ 4 /$. Yet the choice of this parameter only has marginal influence on the result of the identification. The forgetting factor however can significantly influence the result of the identification. For the presented identification algorithm, a factor $\lambda=0,998$ was chosen. The initial value for the parameter vector $\boldsymbol{\theta}(0)$ was set to $\theta_{i}=1$ for each element.

The actual system parameters of the hydraulic system $K^{\prime}, V_{0}, q_{v, \text { nom }}$ and $y_{0 v}$ can then be calculated from the parameter vector as follows:

$$
\begin{gathered}
Q_{v, \text { nom }}=-\sqrt{\Delta p_{\text {nom }}} \cdot \theta_{1} \\
K^{\prime}=-\frac{A_{A}}{\theta_{3}} \\
V_{0}=-\theta_{2} \cdot K^{\prime} \\
y_{0 v}=\frac{\sqrt{\Delta p_{\text {nom }}}}{Q_{v, \text { nom }}} \cdot \theta_{4}
\end{gathered}
$$




\subsection{The valve dynamics subsystem}

The system equation for the valve dynamics subsystem from equations (3) and (4) is:

$$
\frac{1}{\omega_{0 v}^{2}} \ddot{y}_{v}+\frac{2 D_{v}}{\omega_{0 v}} \dot{y}_{v}+y_{v}=K_{v} \cdot U_{v}\left(t-T_{\text {dead }}\right)
$$

The unknown system parameters are the natural frequency $\omega_{0 v}$, the damping coefficient $D_{v}$, the valve gain $K_{v}$ and the dead time $T_{\text {dead }}$. This equation though, cannot be transformed to meet the requirements of the above described parameter estimation that was applied to the hydraulic subsystem (see eq. 8). In Addition, an identification in the time domain would require the valve position $y_{v}$ as well as two more derivatives $\left(\dot{y}_{v}\right.$ and $\left.\ddot{y}_{v}\right)$. With the technically inevitable signal noise, those signals cannot be calculated for the high dynamic of the servo valve.

Therefore an identification within the frequency domain was implemented. This requires a multi-step identification with the determination of the frequency response from measured data that is followed by the parameter estimation. This identification cannot be implemented as an online process. It contains the following steps:

1) Employ a sinusiodial test signal with a frequency $f_{p}$ to the servo valve

2) Calculate real and imaginary part of the frequency response with an orthogonal correlation

3) Calculate Magnitude and Phase shift for the current frequency

4) Repeat steps 1-3 for different frequencies in order to obtain the complete frequency response with magnitude and phase shift

5) Apply a parameter estimation on the magnitude and identify the parameters $\omega_{0 v}, D_{v}$, and $K_{v}$

6) Apply a parameter estimation on the phase shift and identify the last parameter $T_{\text {dead }}$.

The frequency response with magnitude and phase shift can be determined with the help of an orthogonal correlation, which is the "most important frequency response measurement technique for linear systems" (/4/, p. 134). Therefore a sinusoidal signal at a fixed frequency is applied to the servo valve. As the valve is equipped with an onboard position control for the spool, the current position can be measured. From the applied voltage as test signal and the returned spool position, the magnitude and phase shift for the current frequency can be obtained. Figure 6 shows the measured signals. The phase shift and magnitude can clearly be seen and could also be measured and calculated manually.

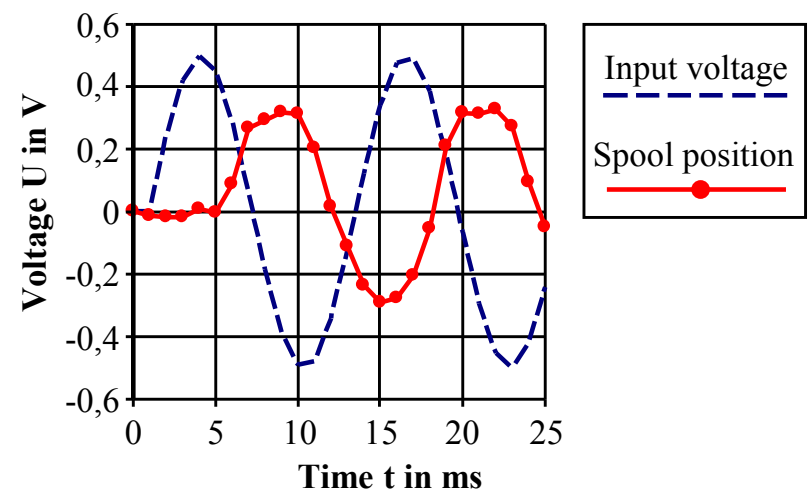

Figure 6: Input voltage and spool position for the servo valve $\left(U_{\max }= \pm 10 \mathrm{~V}\right)$, measurements

The algorithm is based on the orthogonality relations of trigonometric functions. Its main arithmetic operations are the multiplication of the input and output signal followed by an integration. This leads to the imaginary and real part $\operatorname{Im}[G(j \omega)]$ and $\operatorname{Re}[G(j \omega)]$ of the frequency response for the current test signal frequency $f_{p}$ and amplitude $U_{0}$. The orthogonal correlation is an ongoing algorithm over $n$ full periods where the accuracy of the calculated frequency response increases with the number of periods integrated. Detailed description on this method can be found in $/ 4 /$. The equations to determine the frequency response are as follows:

$$
\begin{aligned}
& \operatorname{Re}[G(j \omega)]=\frac{2}{U_{0}^{2}} \cdot \frac{f_{p}}{n} \cdot \int_{0}^{n \cdot \frac{1}{f_{p}}} U_{0} \sin (\omega t) \cdot y_{v} d t \\
& \operatorname{Im}[G(j \omega)]=\frac{2}{U_{0}^{2}} \cdot \frac{f_{p}}{n} \cdot \int_{0}^{n \cdot \frac{1}{f_{p}}} U_{0} \cos (\omega t) \cdot y_{v} d t
\end{aligned}
$$

The correlation should not start before the system reached its steady state. Furthermore, the accuracy increases the longer the integration is calculated. Figure 7 shows the values of the real and imaginary part for a measurement at a test signal frequency of $f_{p}=20 \mathrm{~Hz}$. Both values tend towards a static value after less than 0.5 seconds. This time is even lower for higher frequencies.

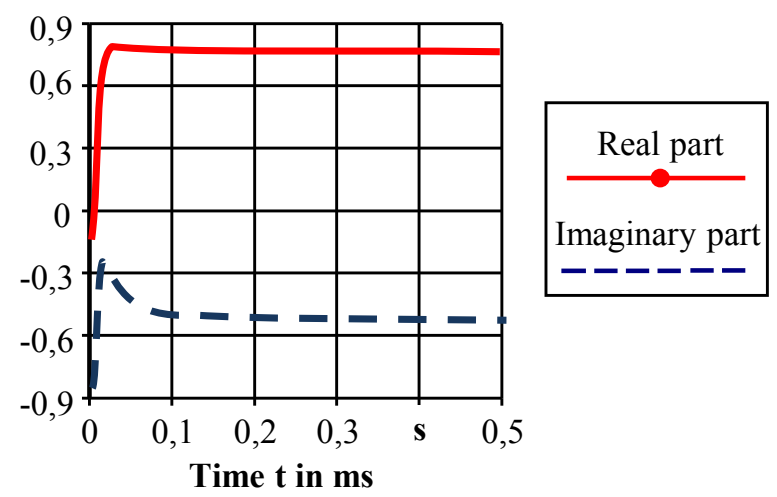

Figure 7: Real- and imaginary part for a test signal frequency $f_{p}=20 \mathrm{~Hz}$ 
Magnitude $|G(j \omega)|$ and phase shift $\varphi$ can then be calculated from the orthogonal correlation, see equations 16 and 17:

$$
\begin{gathered}
|G(j \omega)|=\frac{K_{v}}{\sqrt{\left(1-\frac{\omega^{2}}{\omega_{0 v}^{2}}\right)^{2}+\left(2 D_{v} \cdot \frac{\omega}{\omega_{0 v}}\right)^{2}}} \\
\varphi=-T_{\text {dead }} \cdot \omega-\arctan \left(\frac{2 D_{v} \frac{\omega}{\omega_{0 v}}}{1-\frac{\omega^{2}}{\omega_{0 v}^{2}}}\right)
\end{gathered}
$$

The system behavior of this second order transfer function in the frequency domain can now be transformed to a parameterlinear equation, as demanded in eq. (5). This leads to the following equations for the magnitude at each frequency $\omega$ :

$$
\begin{aligned}
& \gamma_{\text {gain }}=\boldsymbol{\theta}_{\text {gain }} \cdot \boldsymbol{u}_{\text {gain }} \\
& \gamma_{\text {gain }}=|G(j \omega)|^{2} \\
& \boldsymbol{\theta}_{\text {gain }}=\left(\begin{array}{l}
\theta_{1, \text { gain }} \\
\theta_{2, \text { gain }} \\
\theta_{3, \text { gain }}
\end{array}\right) \\
& \theta_{1, \text { gain }}=\frac{2-\left(2 \cdot D_{v}\right)^{2}}{\omega_{0 v}^{2}} \\
& \theta_{2, \text { gain }}=\frac{1}{\omega_{0 v}^{4}} \\
& \theta_{3, \text { gain }}=K_{v}^{2} \\
& \boldsymbol{u}_{\text {gain }}=\left(u_{1, \text { gain }} u_{2, \text { gain }} u_{3, \text { gain }}\right) \\
& u_{1, \text { gain }}=\omega^{2} \cdot|G(j \omega)|^{2} \\
& u_{2, \text { gain }}=-\omega^{4} \cdot|G(j \omega)|^{2} \\
& u_{3, \text { gain }}=1
\end{aligned}
$$

Applying the above described algorithm of the parameter estimation on equations 18 to 21 , the valve parameters $\omega_{0 v}$, $D_{v}$, and $K_{v}$ can be identified. Only the parameter $T_{\text {dead }}$ remains unknown. It can be identified from the phase shift $\varphi$ :

$$
\begin{aligned}
& \gamma_{\text {phase }}=\theta_{\text {phase }} \cdot u_{\text {phase }} \\
& \gamma_{\text {phase }}=\varphi+\arctan \left(\frac{2 D_{v} \cdot \frac{\omega}{\omega_{0 v}}}{1-\frac{\omega^{2}}{\omega_{0 v}^{2}}}\right) \\
& \theta_{\text {phase }}=-T_{\text {dead }} \\
& u_{\text {phase }}=\omega
\end{aligned}
$$

\section{Practical implementation on the drive controller}

The processing power of the controller and the available signal quality are the key points for the practical implementation. The identified system sets its characteristic requirements towards high signal quality and sampling time.

\subsection{Signal quality}

The noise on the measured signals is a major problem during the practical implementation of the identification algorithm. Due to electromagnetic interferences, the existing signal quality is rather low. Mainly the frequency converters and electric motors induce a significant noise level on the machine signals. The identification of the valve dynamics does only require the movement of the valve spool. Therefore the frequency converters and electrical motors are completely switched off and only marginal noise occurs such that no additional filters are necessary.

However, for the identification of the hydraulic system, the deep drawing press needs to be in full operation where high electromagnetic interferences and signal noise occur. The machine signals are filtered and the derivatives are calculated as symmetrical derivative over several measure points. The aim was to retrieve an acceptable signal quality while at the same time retaining the dynamic of the signal with a reasonable effort and complexity towards the applied filter. The chosen signal filter is a moving-average filter (see /8/ for further information). More complex filter algorithms may offer a better filter behavior, especially in the frequency domain, but the moving average filter offers best usability for the chosen application. It can easily be implemented within the machine controller as well as connected to the identification algorithm while at the same time delivering sufficient results for the time domain encoded and processed signal. For every time step $k$, the filter calculates the average over a set number of measure points. Equation 26 shows the moving average filter for a width of $M=5$. The equation for the symmetrical derivative is given in eq. 27 for a width of 5 . The effect of a rising filter width is shown in figure 8 . The equations are formulated for an offline-processing in order to reduce the time delay. For the practical implementation on the controller this requires to save $(M+1)$ data points where the filtered data is calculated with a time delay of $\left[0,5 \cdot(M+1) \cdot T_{a}\right]$. This needs to be applied to all measured signals in order to retain synchronism.

$$
\begin{gathered}
y_{f i l t, k}=\frac{y_{k+2}+y_{k+1}+y_{k}+y_{k-1}+y_{k-2}}{5} \\
\dot{y}_{f i l t, k}=\frac{y_{k+2}+y_{k+1}-y_{k-1}-y_{k-2}}{6}
\end{gathered}
$$




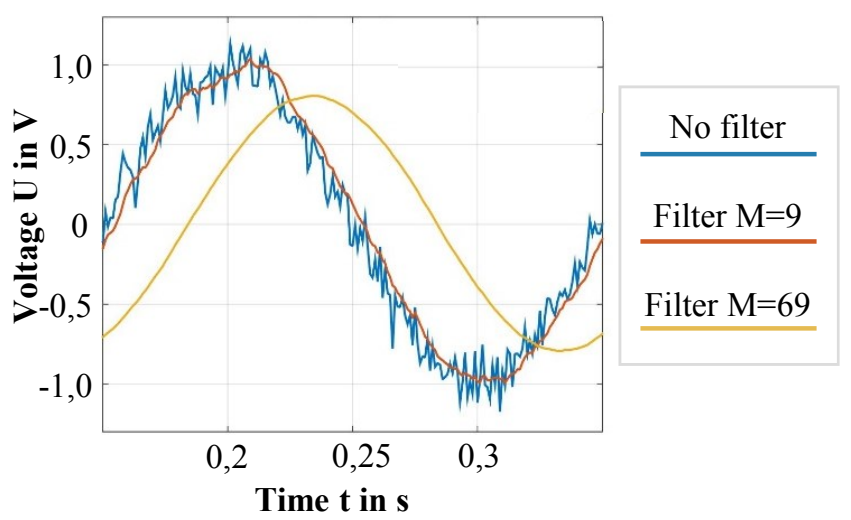

Figure 8: Effect of different filter parameters on the signal processing

In order to determine the optimal filter parameters, a simulation model of the hydraulic system was used together with a model of the occurring noise. With the help of the simulation model, a noise-free reference could be found for the identification. The filter parameters could be optimized in a way that the identification result with noise and the result without noise were compared for best results. The method is shown in Figure 9:

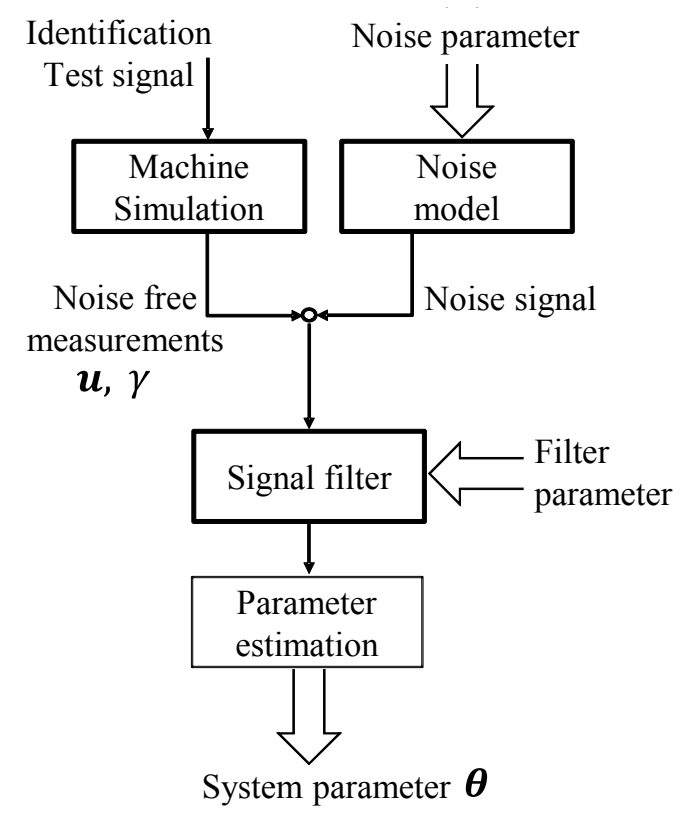

Figure 9: Method for finding optimal filter parameters

The machine simulation is a verified system model of the deep drawing press from previous research activities (see /9/ and $/ 10 /$ ). The noise model was generated from analyzing the actual occurring noise as seen in figure 10. Signal noise can be considered as white noise for most applications $/ 11 /$. Therefore the measured signal noise was evaluated. Its deviation is similar to a normal distribution, though it does not pass the Chi-Squared-Test. This can be explained as there are specific frequencies that occur more frequent, such as at $50 \mathrm{~Hz}$ and $100 \mathrm{~Hz}$. Nevertheless, most frequencies are evenly distributed and the parameters for a normal distributed white noise signal can be obtained with reasonable effort. The corresponding parameters of an ideal white noise signal for the current test setup are given in table 1 . The median $\mu$ is zero for all measured signals and the standard deviation $\sigma$ differs due to the different gain factors for the analog signal processors.

Table 1: noise parameters for the modelled white noise signal with normal distribution

\begin{tabular}{|c|c|c|c|}
\hline & Pressure & Position & Valveposition \\
\hline$\mu$ & $0 \mathrm{~Pa}$ & $0 \mathrm{~m}$ & $0 \%$ \\
\hline$\sigma$ & $2,2 \times 10^{5} \mathrm{~Pa}$ & $1,2 \times 10^{-4} \mathrm{~m}$ & $6,8 \times 10^{-3} \%$ \\
\hline
\end{tabular}
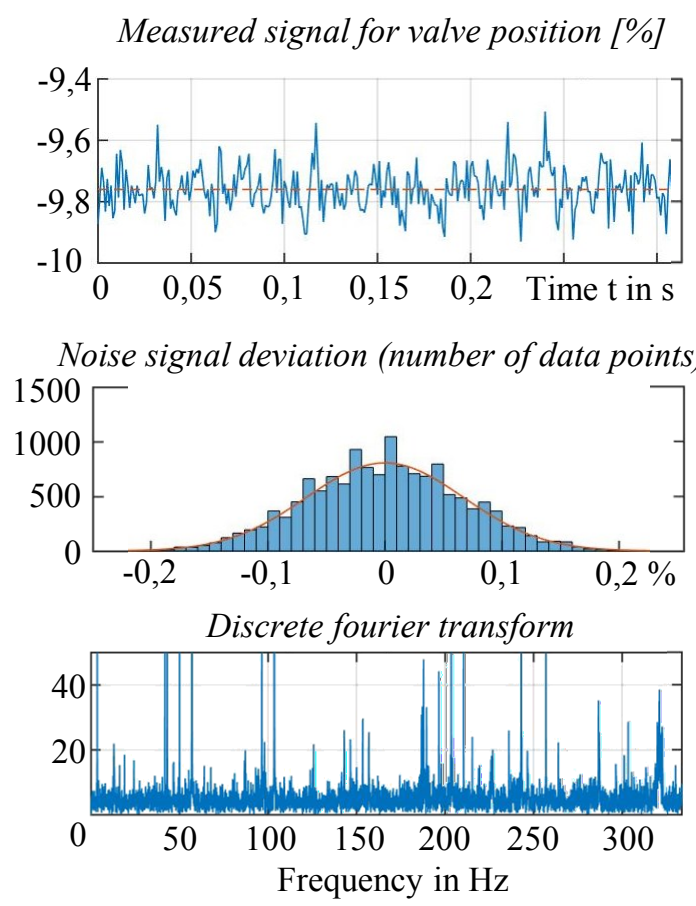

Figure 10: noise on the valve position signal and the occurring deviation

The quality of the filter will be evaluated by computing 150 parameter estimations with the added noise model with one set of filter parameters. Due to the chosen random noise generator, the estimated parameters $\theta_{i}$ will result in a different result for every single simulation. The bias and standard deviation of all 150 estimation results will be used to evaluate the quality of the chosen filter parameters. Figure 11 shows the deviation of the system parameter $\theta_{3}$ for 150 simulation with the noise parameter as shown in table 1 and no filter applied. All values are normalized. The mean value of the identification result is at $6 \%$ of the value without noise (Bias) and the standard deviation within the 150 simulations is at $48 \%$ (interval from $-42 \%$ to $54 \%$ ). This means that the parameter estimation does not deliver reliable results. 


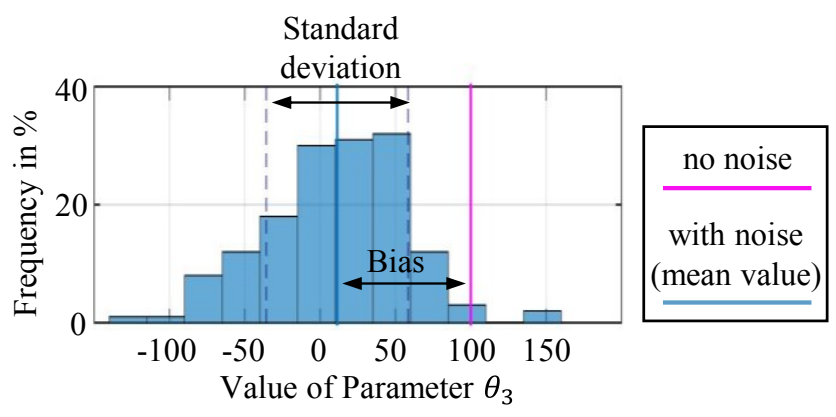

Figure 11: Bias and Standard deviation for $\theta_{3}$ without filter

This process is repeated for a different set of filter parameters from $M=0 \ldots 50$ for the moving average filter as well as for the symmetrical derivative with 150 simulations for each filter. The bias of the identified system parameters for $\theta_{1 \ldots 3}$ is shown in figures 12 to 14 in dependence from the filter parameters for a time step of the machine controller of $T_{a}=2 \mathrm{~ms}$. It can be seen that the parameter $\theta_{1}$ delivers best results with a maximum relative deviation of $0,702 \%$. This is because there is no signal derivative necessary to calculate its value:

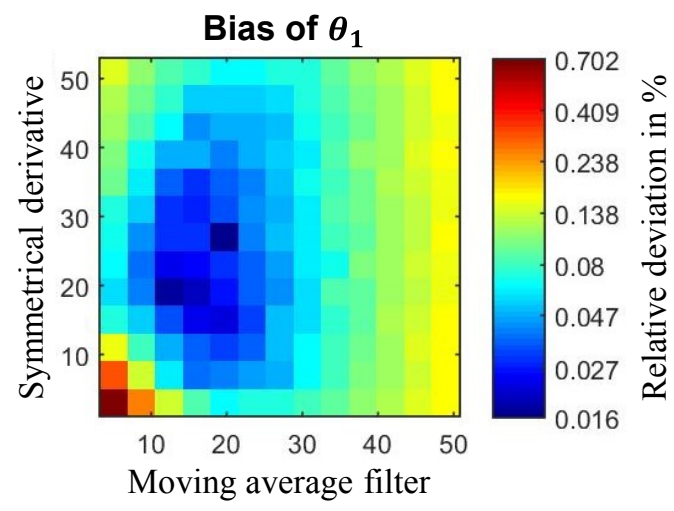

Figure 12: Bias of $\theta_{1}$ for different numbers of data points for the moving average filter and the symmetrical derivative

Both other parameters $\theta_{2,3}$ show a clear dependence. For $\theta_{2}$ the number of data points on the symmetrical derivative and the moving average filter should be similar and even a high filter parameter still delivers good results.

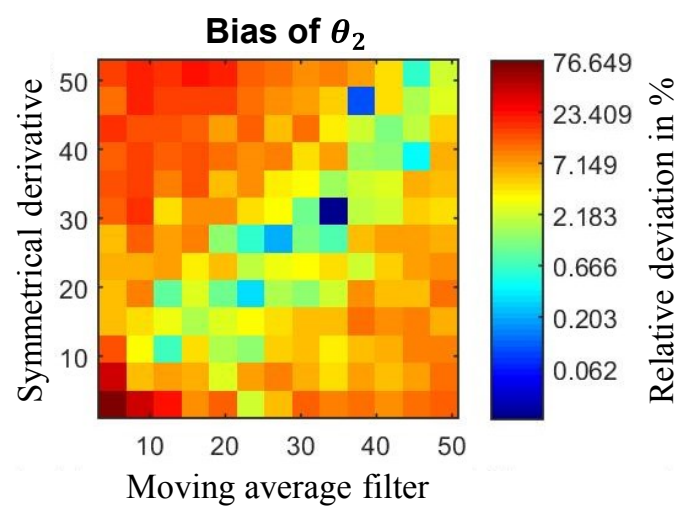

Figure 13: Bias of $\theta_{2}$ for different numbers of data points for the moving average filter and the symmetrical derivative
However, the accuracy of parameter $\theta_{3}$ decreases with rising filter parameters. The optimum for the filter parameters can be found at about $M=20 \ldots 30$ for the moving average filter as well as for the symmetrical derivative.

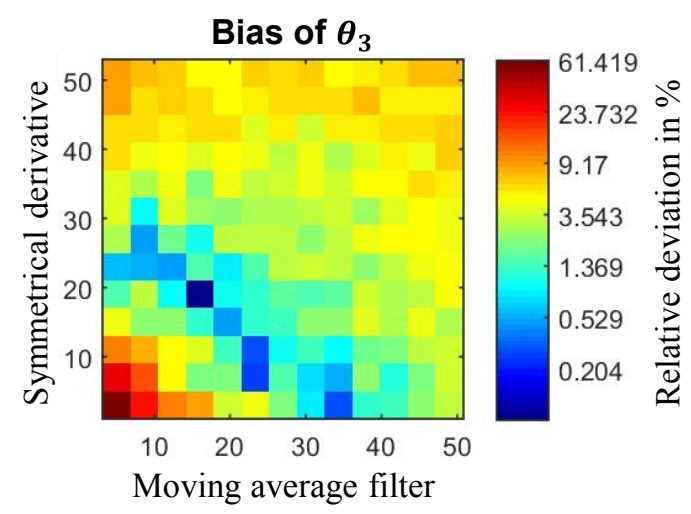

Figure 14: Bias of $\theta_{3}$ for different numbers of data points for the moving average filter and the symmetrical derivative

The standard deviation shows a similar behavior for all identification parameters. The value decreases monotonous with rising filter width, as shown in figure 15.

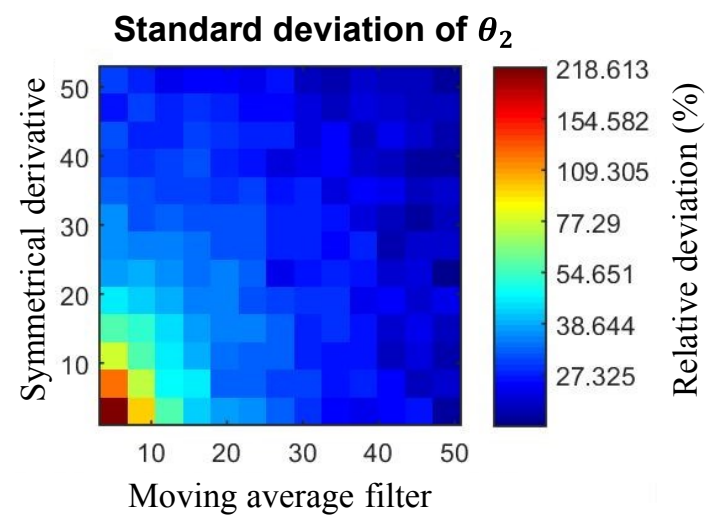

Figure 15: Standard deviation for $\theta_{2}$ for different numbers of data points for the moving average filter and the symmetrical derivative

\subsection{Sampling time}

A constant sampling time of the machine controller is essential for the algorithm as all signal derivations are calculated within the algorithm. The Nyquist-Shannon sampling theorem states that the measuring frequency theoretically should be double the highest frequency content of the signal. Otherwise, aliasing would occur. The identification algorithms requires a signal that includes no less than the highest system frequency, otherwise the system cannot be identified correctly. Each of the subsystems has its own dynamic and for each identification, the sampling time must be low enough to measure this dynamic and additionally to allow for sufficient filtering in order to suppress noise. For the valve dynamics subsystem the limiting frequency is the eigenfrequency of the valve, respectively the highest applied test signal frequency. The hydraulics subsystem can be 
characterized by means of a step response test and the $t_{63}$ time constant for an assumed PT1 first-order lag element. The $t_{63}$ time constant was derived from a sample step response test at the machine. With a step function on the valve, the pressure response was measured. The $t_{63}$ time constant is when the pressure signal passes $63 \%$ of its final static value. For the assumed first-order lag element, the $t_{63}$ is equivalent to the time constant $T$, respectively the inverse breakpoint frequency $1 / \omega_{B}$. The sampling time should be as low as $0.1 t_{63}$.

The investigated servo-valve has a high eigenfrequency and the test signal frequency goes up to $f=160 \mathrm{~Hz}$, so the sampling time should be at about $T_{a} \approx 1 \mathrm{~ms}$. The time constant of the hydraulic system is at $t_{63} \approx 90 \mathrm{~ms}$ (breakpoint frequency of merely $\omega_{B}=69 \mathrm{~Hz}$ ), sampling time should be no higher than $T_{a}=9 \mathrm{~ms}$. It has to be noted, that the investigated hydraulic deep drawing press has a comparable low ram speed of $v_{\text {ram }}=40 \mathrm{~mm} / \mathrm{s}$. Industrial servo presses feature speeds of up to $300 \mathrm{~mm} / \mathrm{s}$. Experimental data shows that the characteristic time constant for these machines is at $t_{63}=3 \mathrm{~ms}$. The sampling time should be equally lower. With regards to the applied digital signal filters, an even lower sampling time is preferred. Therefore an individual task for signal processing was implemented within the machine controller that runs at $2 \mathrm{~ms}$. The identification algorithm is executed every $10 \mathrm{~ms}$. This leads to sufficient results for the hydraulic subsystem with minimal required processing power. However, the identification for the valve subsystem is calculated in a specific task at $1 \mathrm{~ms}$ to take the high system dynamic into account.

\section{Identification results}

The above described parameter estimation algorithm was successfully implemented on the machine controller of the die cushion in a hydraulic deep drawing press.

The identification of the valve dynamics subsystem is shown in figure 16. A frequency of $(10,20 \ldots 160) \mathrm{Hz}$ at $0.5 \mathrm{~V}(5 \%$ of the nominal valve voltage) was applied at the servo valve and the magnitude and phase shift calculated by means of the orthogonal correlation. Due to the low voltage level, no spool velocity saturation should occur. This also means that the different behavior for different amplitudes is not modelled and the model is only valid for low voltage magnitudes. During identification, no signal filter was applied as all machine drives were switched off and there was no significant signal noise. The parameter estimation algorithm identified the system parameters for the assumed second order system with an additional dead-time (see eq. 13). The system parameters are as follows:

Damping ratio: $D=0.6735$

Natural frequency: $\omega_{0 v}=183.9 \mathrm{~Hz}$

Gain: $K_{v}=1.005$ and

Dead Time: $T_{\text {dead }}=0.00 \mathrm{~s}$
The Bode plot of the identified system model shows a good accuracy in comparison to the measured data (see fig. 16).

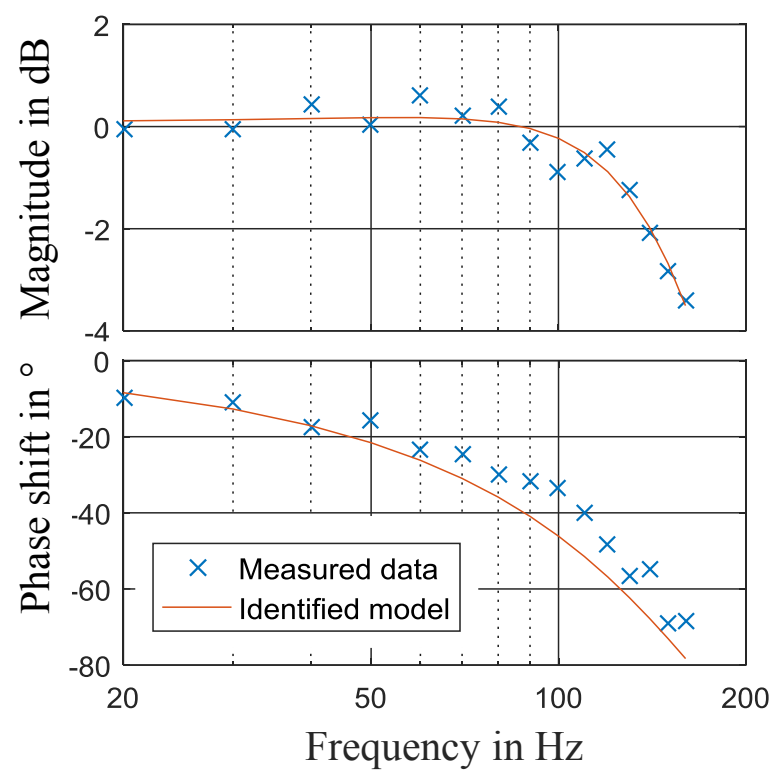

Figure 16: Magnitude and Phase shift for the identified servo valve in in the deep drawing press

The identification of the hydraulic subsystem is more difficult, as it sets higher standards towards signal quality and correct implementation of a signal filter. With the applied filter, the identification algorithm delivers good results. Figure 17 shows the identification of the bulk modulus over time from the machine controller with the implemented recursive least square identification algorithm. It can be seen that it approaches towards a constant end value. The parameters were as follows:

Sampling time (signal processing): $t_{S}=2 \mathrm{~ms}$

Sampling time (identification): $t_{i}=10 \mathrm{~ms}$

Moving average filter (width): $b=31$

Symmetrical derivative (width): $d=25$

Forgetting factor: $\lambda=0,998$

Parameter initial values: $\theta_{1 \ldots 4}=1$

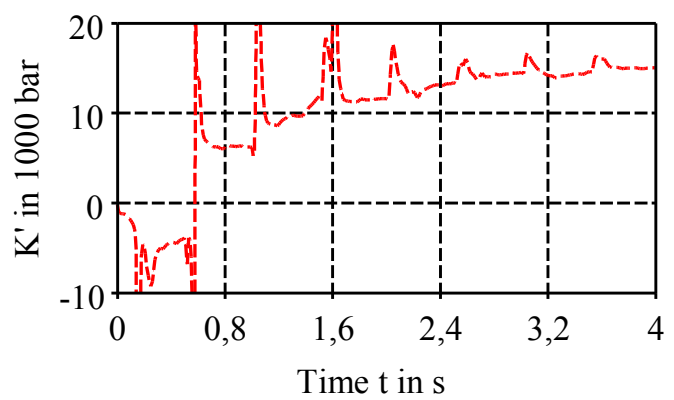

Figure 17: Identification of the bulk modulus 
The identification result and the speed at which it approaches towards the static value can be improved by setting initial values as a "good guess". However, the algorithm still shows good results at a reasonable speed even with the defined initial values being set at ' 1 '.

The identification algorithm was executed numerous times in order to determine its stability and robustness. The identification results for 30 consecutive measures are shown in figure 18 for the nominal volume flow, the dead volume and the bulk modulus. The figure also shows the mean value though all identifications with a solid line. It has to be noted that the oil temperature is not constant throughout all 30 measures, but rises from $26^{\circ} \mathrm{C}$ for the first identification to $32^{\circ} \mathrm{C}$ for the last identification. This explains the characteristic of the nominal volume flow $Q_{v, \text { nom }}$ due to changing properties of the hydraulic fluid.
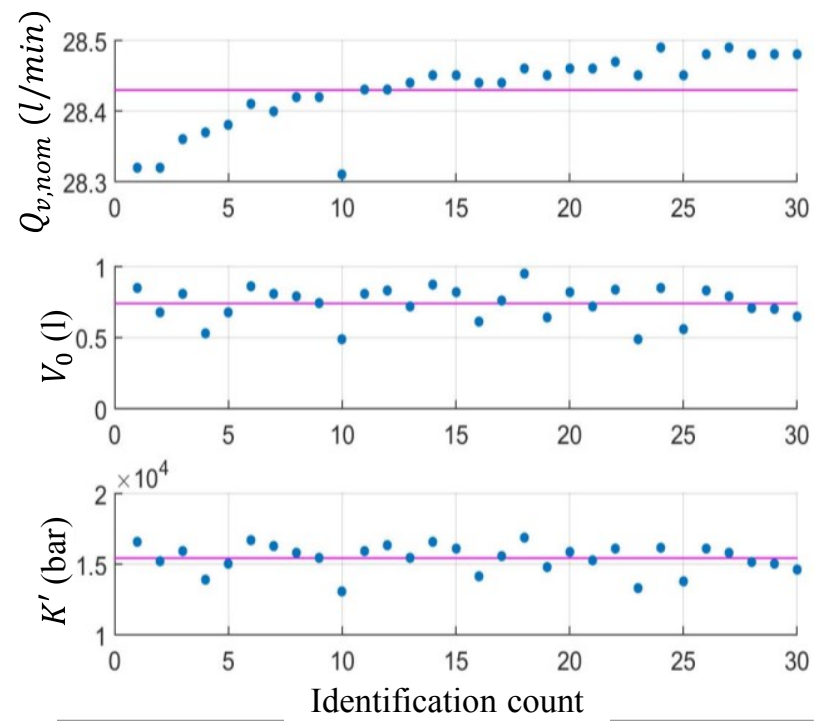

Figure 18: Identification results for 30 consecutive experiments

\section{Outlook}

The paper shows the successful implementation of an identification algorithm within the machine controller of an industrial type hydraulic deep drawing press. This offers a high potential for further applications to reduce manual maintenance and increase the performance of the hydraulic drive. The online-identification algorithm is able to detect changing system parameters that can be used for condition monitoring, predictive maintenance or fault diagnosis. The described method can further be applied for other hydraulic systems and is not limited to the deep drawing press. In addition, the now fully identified system model can be used for controller design. The implementation of a feed-forward element based on the volume flow characteristics of the servo valve is a common method to improve the control quality of hydraulic die cushion drives [12] and the system parameters $Q_{v, n o m}, \Delta p_{n o m}$ and $y_{0}$ could significantly improve the system behavior. Experimental results for the control behavior of the die cushion that was described in this paper are shown in Figure 19.

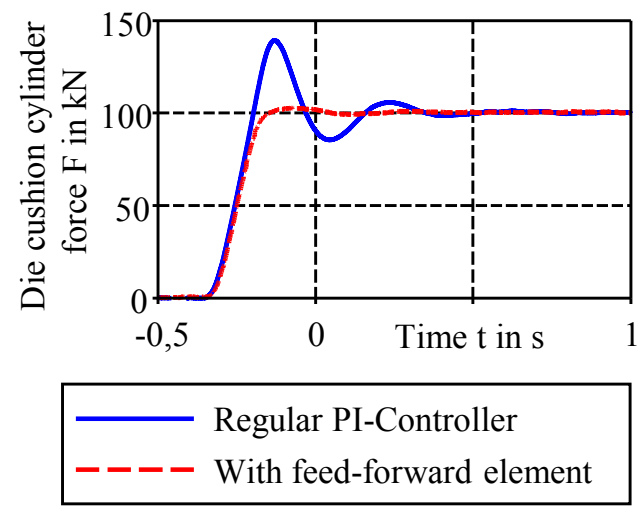

Figure 19: Control accuracy of the die cushion

\section{Acknowledgements}

The presented research is part of the project KF2453605LP3 - "Entwicklung eines neuartigen adaptiven Regelsystems zur automatisierten und selbstoptimierten Inbetriebnahme hochdynamischer elektrohydraulischer Pressen mit integrierter Parameternachführung im laufenden Betrieb", which is funded by the Federal Ministry for Economic Affairs and Energy, Germany within the ZIM program.

\section{Supported by:}

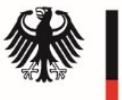

Federal Ministry

for Economic Affairs and Energy

on the basis of a decision

by the German Bundestag 


\section{References}

[1] Lohse, Harald; Helduser, Siegfried; Marthiens, Olaf; Matthias, Thorsten; Behrens, Bernd-Arno (2010): Reglerauslegung für hydraulische Tiefziehpressen Unterstützt durch ganzheitliche Simulation. In: $\mathrm{O}+\mathrm{P}$ Ölhydraulik und Pneumatik 2010 (3), S. 68.

[2] Helduser, Siegfried (2006): Elektrisch-hydraulische Systemtechnik. Entwicklungsschwerpunkte in der Stationärhydraulik. In: O+P Ölhydraulik und Pneumatik 2006 (1), S. 16-23.

[3] Schoppel, Georg (2003): Beiträge zur automatischen Inbetriebsetzung und Regelung hydraulischer Zylinderantriebe. Aachen: Shaker.

[4] Isermann, Rolf (2011): Identification of Dynamic Systems, Springer.

[5] Keesmann, K. J. (2011): System identification an introduction, Advanced textbooks in control and signal processing, Springer.

[6] Ljung, L. (2009): System Identification. Theory for the user, Prentice Hall.

[7] Jelali, M.; Kroll, A. (2004): Hydraulic servo-systems. Modelling, identification and control. Springer London Ltd.

[8] Orfanidis, S. J. (1996): Introduction to signal processing, Prentice Hall signal processing series. Prentice Hall

[9] Schulze, T.; Weber, J.; Penter, L.; Großmann, K. (2014): Modelling and Simulation of the die cushion in a hydraulic deep drawing press. $16^{\text {th }}$ ITI Symposium, Dresden

[10] Schulze, T.; Weber, J.; Großmann, K.; Penter, L.; Schenke, C. (2015): Hydraulic die cushions in deep drawing presses - analysis and optimization using coupled simulation. ASME/BATH 2015 Symposium on Fluid Power and Motion Control, Chicago

[11] Smith, S. W. (1999): The scientist and engineer's guide to digital signal processing. California Technical Publication

[12]Helmke, M.; Majer, H.; Thanassakis, A. (2016): Improvement of hydraulic control quality for deep drawing presses through retrofit. $10^{\text {th }}$ IFK 2016, Dresden, 